\title{
Autism and autistic traits in those who died by suicide in England
}

Sarah Cassidy, Sheena Au-Yeung, Ashley Robertson, Heather Cogger-Ward, Gareth Richards, Carrie Allison, Louise Bradley, Rebecca Kenny, Rory O'Connor, David Mosse, Jacqui Rodgers and Simon Baron-Cohen

\section{Background}

Autism and autistic traits are risk factors for suicidal behaviour.

\section{Aims}

To explore the prevalence of autism (diagnosed and undiagnosed) in those who died by suicide, and identify risk factors for suicide in this group.

\section{Method}

Stage 1: 372 coroners' inquest records, covering the period 1 January 2014 to 31 December 2017 from two regions of England, were analysed for evidence that the person who died had diagnosed autism or undiagnosed possible autism (elevated autistic traits), and identified risk markers. Stage 2: 29 follow-up interviews with the next of kin of those who died gathered further evidence of autism and autistic traits using validated autism screening and diagnostic tools.

\section{Results}

Stage 1: evidence of autism (10.8\%) was significantly higher in those who died by suicide than the $1.1 \%$ prevalence expected in the UK general alive population (odds ratio $(\mathrm{OR})=11.08,95 \% \mathrm{Cl}$ 3.92-31.31). Stage 2: 5 (17.2\%) of the follow-up sample had evidence of autism identified from the coroners' records in stage 1. We identified evidence of undiagnosed possible autism in an additional 7 (24.1\%) individuals, giving a total of 12 (41.4\%); significantly higher than expected in the general alive population (1.1\%) $(\mathrm{OR}=19.76,95 \% \mathrm{Cl} 2.36-165.84)$. Characteristics of those who died were largely similar regardless of evidence of autism, with groups experiencing a comparably high number of multiple risk markers before they died.

\section{Conclusions}

Elevated autistic traits are significantly over-represented in those who die by suicide.

\section{Keywords}

autism spectrum disorders; autistic traits; suicide; mortality; autism.

\section{Copyright and usage}

(c) The Author(s), 2022. Published by Cambridge University Press on behalf of the Royal College of Psychiatrists. This is an Open Access article, distributed under the terms of the creative Commons Attribution licence (https://creativecommons.org/ licenses/by/4.0/), which permits unrestricted re-use, distribution, and reproduction in any medium, provided the original work is properly cited.

\section{Background}

Autism is a lifelong neurodevelopmental condition consisting of difficulties in social communication, adapting to unexpected change, heightened sensory sensitivity and restricted interests. ${ }^{1}$ There are many barriers to obtaining an autism diagnosis, such as knowledge of autism, availability of diagnostic services and funding. ${ }^{2}$ A population study in the UK screened the adult population, including individuals with previously unknown or undiagnosed autism, to establish the true prevalence of autism, and found that approximately $1.1 \%$ of UK general population adults meet criteria for an autism diagnosis. ${ }^{3}$ Those with elevated autistic traits can experience similar difficulties to autistic people, such as sensory hypersensitivity, and social communication and sensorimotor difficulties. ${ }^{4}$ Many adults who are high in autistic traits, and in particular women, are likely to go undiagnosed, even though they might meet criteria for an autism diagnosis owing to lack of appropriate services, validated diagnostic tools and a tendency to camouflage their autistic behaviours and characteristics to 'fit in' in social situations. ${ }^{2}$ Previous research has confirmed an increased vulnerability to mental health problems in both autistic people and those with elevated autistic traits. ${ }^{5,6}$

Autistic people are at significantly higher risk of suicidal thoughts and behaviours compared with non-autistic people. ${ }^{5-8}$ Lifetime prevalence of suicidal ideation in autistic adults ranges between $19.7 \%$ and $66 \%$, and suicide attempts between $1.8 \%$ and $36 \%,{ }^{5}$ the highest prevalence estimates being in late-diagnosed adults. ${ }^{7}$ Self-reported autistic traits in those without autism diagnoses are also associated with increased risk of suicidal thoughts and behaviours. $^{5,8}$
Research has found that autism and autistic traits are over-represented in groups at risk for suicide. A high proportion of patients with depression $(11 \%)^{9}$ and women with borderline personality disorder $(15 \%)^{10}$ meet diagnostic criteria for co-occurring autism. Suicide attempts were found to be highest in people with a co-occurring diagnosis, across both groups. A total of $45 \%$ of women with elevated autistic traits in the region of clinical concern for possible autism reported making a suicide plan, and $16 \%$ had attempted suicide. ${ }^{11}$ A study found that $40.6 \%$ of those who attempted suicide scored above the cut-off for clinical concern for possible autism on the Autism Spectrum Quotient, ${ }^{12}$ even after removing those with a self-reported diagnosis or suspected autism from the analysis. ${ }^{13}$

Studies have shown significantly increased risk of dying by suicide in autistic people compared with the general population. ${ }^{14,15}$ As discussed above, many autistic people remain undiagnosed, with increased risk of attempting suicide. However, no studies have yet explored potentially undiagnosed autism or attempted to quantify autistic traits in those who have died by suicide.

Autism and autistic traits are beginning to be considered for inclusion in contemporary suicide theories. ${ }^{5}$ For example, cognitive inflexibility may reduce ability to solve problems in stressful or challenging circumstances, and therefore impair the ability to see a way out, increasing the risk of experiencing entrapment, with suicide being perceived as the only possible escape route. ${ }^{5,16}$ Autistic people and those with elevated autistic traits report camouflaging their autistic behaviours in an attempt to 'fit in' in social sitations, ${ }^{17}$ leading to feelings of not being accepted for one's true self in society (termed 'thwarted belongingness'), increasing their risk of 
suicidality. ${ }^{18,19}$ Autistic people's increased vulnerability to adverse life events, such as bullying, abuse, exploitation, social exclusion and poverty, also increases their risk of suicidality. ${ }^{6}$ Autistic people (diagnosed and undiagnosed), are also more likely to experience perseverative suicidal thoughts, and impulsively attempt suicide without a plan during a crisis when means for self-harm were present, which could particularly increase their risk of death by suicide. ${ }^{20,21}$

\section{Aims}

Previous general population studies have used psychological autopsy methods to gather evidence of diagnoses, characteristics and risk markers in those who died by suicide. Information is gathered from a variety of sources, such as coroners' inquest records, medical records and interviews with next of kin. ${ }^{22,23}$ The psychological autopsy method can be used to systematically gather evidence in support of previously unidentified diagnoses (such as autism), personality traits (such as autistic traits), and unique characteristics and risk markers for suicide in different groups (such as those with and without evidence of autism). This approach can therefore identify unique suicide prevention targets in different groups. ${ }^{22}$ The current study is particularly novel as it utilises a large analysis of coroners' inquest records, followed by psychological autopsy interviews with a subsample of next of kin of those who died. Through these two stages, we address the following research questions.

(a) What is the prevalence of autism and evidence for autistic traits in those who died by suicide in England?

(b) What are the characteristics and risk markers for suicide in those who died by suicide who had evidence of autism and/or elevated autistic traits compared with those who died by suicide who had no evidence of autism?

We predicted that autism, and elevated autistic traits (indicating possible undiagnosed autism), would be significantly over-represented in those who died by suicide compared with the prevalence expected in the general alive population.

\section{Method}

\section{Design}

Recruitment to the two stages of the study is shown in Fig. 1. In stage 1, coroners' inquest records covering dates of death between 1 January 2014 and 31 December 2017 were requested from two regions of England.

Data were obtained from the Office of National Statistics 2011 census (https://www.ons.gov.uk/census/2011census) and 20162018 Public Health England Suicide Prevention Profile (https:// www.gov.uk/government/statistics/suicide-prevention-profile-updates) for each site. Site 1: 77.4\% employed, 97.5\% White (2011 census), prevalence of suicide 8.4 per 100000 (2016-2018); site 2: $81 \%$ employed, 90.3\% White (2011 census), rate of suicide $8.8-12.2$ (across different parts of the region) per 100000 (2016-2018). In the rest of the UK: $75 \%$ employed, $85.4 \%$ White (2011 census), rate of suicide 9.6 per 100000 (2016-2018).

We independently identified records where the person likely died by suicide and analysed these records for: (a) evidence of autism; and (b) characteristics of those who died. The data entry for stage 1 took place between October 2015 and July 2019. Data entry was undertaken by S.C., L.B., A.R., S.A.-Y. and G.R. In stage 2, interviews were conducted with next of kin recruited through the coroners' offices included in stage 1 to gather evidence of autism for those who died.

The interviews for stage 2 took place between June 2018 and March 2019. Interviews were undertaken by S.C., A.R., S.A.-Y.and H.C.-W.

\section{Ethical approval}

Ethical approval was granted by the relevant local ethics committee at Coventry University (stage 1: P42264; stage 2: P60970). Our ethical approval stipulates that the sites included in this study are not published to protect the anonymity of those who died and their families.

\section{Stage 1: analysis of coroners' inquest records}

Evidence of suicide

Coroners' inquests covering dates of death between 1 January 2014 and 31 December 2017 returning a conclusion of suicide, narrative,

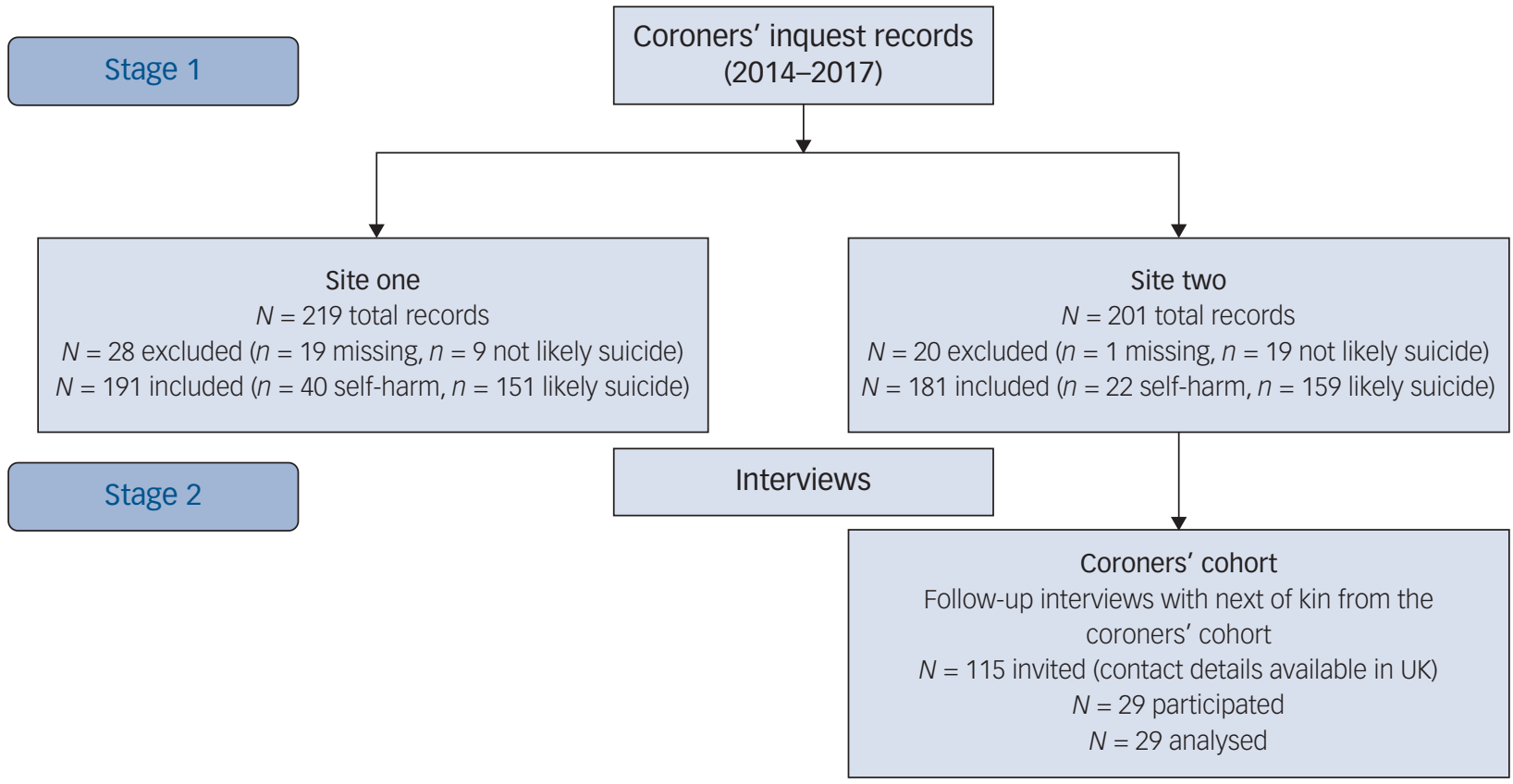

Fig. 1 Recruitment to stage 1 and stage 2 of the study. 
open or other suspected suicide (for example drug and alcohol related, accident/misadventure) were requested from coroners' offices in two regions of England.

In 2019, the standard of proof for a suicide conclusion in all coroners' inquests changed from a criminal standard (beyond reasonable doubt) to a civil standard (balance of probabilities). ${ }^{24}$ The coroners' inquests analysed in the current study therefore used the criminal standard of proof to determine a conclusion of suicide. This criminal standard of proof in coroners' inquests can underestimate the true rate of suicide, ${ }^{25}$ and conclusions for people who likely died by suicide also vary considerably among coroners. ${ }^{26}$ Hence, we independently reviewed all coroners' inquests to determine whether the person likely died by suicide, as recommended by best practice guidelines for psychological autopsy studies. ${ }^{23}$

Cause of death was coded as:

(a) self-harm, using the ICD- $10^{27}$ definition of intentional selfharm where intent to end life is undetermined; or

(b) suicide, where there was evidence of intentional self-harm with intent to end life, such as leaving a note or message, communication of suicidal intent to others, previous suicide attempts, or the method and/or circumstances of the suicide that suggested suicidal intent (i.e. that the person who died could have reasonably have predicted that the method of self-harm would have likely resulted in their death).

A total of 372 coroners' inquest records were included in the analysis (Fig. 1). Of these 98 (26.3\%) were independently reviewed for evidence of self-harm/suicide by two members of the research team who were also responsible for inputting the data from the coroners' records (S.C., L.B., G.R., A.R., S.A.-Y.). Of the 98 selected for independent review, $32(32.7 \%)$ of the sample had evidence of possible autism, $58(59.2 \%)$ had a suicide conclusion returned by the coroner and $40(40.8 \%)$ had another conclusion returned where the intent was unclear (such as open, alcohol/drug related, misadventure).

There was agreement between the two raters for $86(87.8 \%)$ of the records selected for independent review. Disagreements were resolved with discussion to reach consensus. Of the 372 records included in the analysis, the coroners' returned a conclusion of suicide for 243 individuals (65.3\%), and the research team agreed with the coroners' conclusion of suicide in $100 \%$ of these records. Of the remaining 129 records where the coroners' returned a conclusion indicating likely suicide (for example open, drug/alcohol related, misadventure), the research team coded 67 (51.9\%) as death by likely suicide, and $62(48.1 \%)$ as death by self-harm.

Out of the 372 records, the research team classified 62 (16.7\%) as death by self-harm, and $310(83.3 \%)$ as death by suicide (Table 1$)$.

\section{Evidence of autism}

We devised a checklist to systematically gather evidence of autism from the coroners' inquest records based on the main symptom domains in DSM-5 $5^{1}$ (see Appendix). A total of 95 (25.5\%) of the coroners' inquest records were independently rated for evidence of autism by two members of the research team who were also responsible for inputting the data from the coroners' records (S.C., L.B., G.R., A.R., S.A.-Y.). All records identifying evidence of autism (possible/strong/definite) were checked by a second rater, alongside a random sample of records with no evidence of autism. There was agreement for $81(85.3 \%)$ of records. Disagreements were resolved with discussion to reach consensus.

\section{Risk markers}

A data spreadsheet was developed, informed by previous research, to capture qualitative data pertaining to a broad range of
Table 1 Evidence of autism in cases of likely suicide and self-harm identified from the coroners' inquest records

\begin{tabular}{|c|c|c|}
\hline & $\begin{array}{c}\text { No } \\
\text { evidence }\end{array}$ & $\begin{array}{l}\text { Evidence of } \\
\text { autism }^{\text {a }}\end{array}$ \\
\hline Suicide, $n(\%)(n=310)^{\mathrm{b}}$ & $273(88.1)$ & 37 (11.9) \\
\hline Male, $n(\%)^{c}$ & $211(77.3)$ & $32(86.5)$ \\
\hline Age, years: mean (s.d.) & $47.51(18.2)$ & $44.86(15.5)$ \\
\hline $\begin{array}{l}\text { Self-harm (without clear evidence of } \\
\text { intent), } n(\%)(n=62)^{\mathrm{b}}\end{array}$ & $59(95.2)$ & $3(4.8)$ \\
\hline Male, $n(\%)^{\mathrm{c}}$ & $51(86.4)$ & $2(66.7)$ \\
\hline Age, mean (s.d.) & $42.93(10.9)$ & $37.33(10.1)$ \\
\hline Suicide + self-harm, $n(\%)(n=372)^{\mathrm{b}}$ & $332(89.3)$ & $40(10.8)$ \\
\hline Male, $n(\%)^{\mathrm{c}}$ & $262(78.9)$ & $34(85)$ \\
\hline Age, years: mean (s.d.) & $46.7(17.2)$ & $44.3(15.2)$ \\
\hline \multicolumn{3}{|c|}{$\begin{array}{l}\text { a. Evidence of autism, sum of 'possible diagnosis' + 'strong evidence' + 'definite diag- } \\
\text { nosis'. } \\
\text { b. Denotes percentage calculated by row. } \\
\text { c. Denotes percentages calculated by column. }\end{array}$} \\
\hline
\end{tabular}

characteristics of those who died (Table 2). ${ }^{22,23}$ Content analysis was conducted on these qualitative data to capture the full range of characteristics of each person who died. This method was used as it is more open to discovering new characteristics in the underexplored area of suicide in autism, rather than relying on a pre-existing theoretical framework. S.C. conducted the initial content analysis. A second researcher (H.C.-W.) independently conducted a content analysis on $80(21.5 \%)$ of the coroners' records to ensure that the extracted codes captured the full range of data. Two additional codes were identified by H.C.-W., which S.C. added to the full data-set. To ensure a sufficient sample size in analyses, these codes were collapsed into broader categories (see Table 2 for details of all codes included in the broader categories).

\section{Analysis}

Chi-square analyses compared: (a) the number of individuals with confirmed autism; and (b) total evidence of autism (possible, strong and definite) identified in the coroners' inquest records, to the prevalence of autism expected in the general population. We used the most recent prevalence estimate of autism identified in the UK adult population (1.1\%); this estimate included undiagnosed or unknown cases of individuals with autism, which is therefore similar to the approach taken for the current study. ${ }^{3}$

The frequency of mental and physical health problems, service contact and psychosocial risk markers recorded for those who died with any evidence of autism (possible, strong and definite) was compared with that of those who died with no evidence of autism. Odds ratios (ORs) were calculated as an estimate of effect size. To correct for multiple comparisons in the analysis, a Bonferroni-corrected alpha level of $P<0.0036$ was used. Chi-square analyses were only performed on variables with expected cell counts of at least five, and Fisher's exact tests were used for cell counts between 4 and 4.9.

\section{Stage 2: interviews with next of kin}

\section{Participants}

Next of kin of those who died were invited to take part through the coroners' office of site 2 for all inquests included in stage 1 (Fig. 1). Through the coroners' office, out of the 181 records included in the stage 1 analysis, we were able to invite next of kin of 115 people who died, who were in the UK and for whom contact details were available.

To explore whether the characteristics of those who died were different depending on whether we were able to invite their next of kin to take part in stage 2 or not, we compared age, gender and evidence of autism between these two groups. There were no significant differences in gender $\left(\chi^{2}=0.21, P=0.65\right.$; OR $=1.19,95 \% \mathrm{CI}$ 


\section{Table 2 Characteristics of those who died identified from the coroners' inquest records}

\section{Risk markers}

No evidence $(n=332)$

Evidence of autism ${ }^{\mathrm{a}}(n=40)$

Overall total $(n=372)$

Comparisons (evidence versus no evidence of autism)

Total number of risk markers, ${ }^{\mathrm{b}}$ mean (s.d.)

6.14 (3.18)

6.45 (4.36)

6.17 (3.32)

OR $(95 \% \mathrm{Cl})$

$P$

suicide attempt(s)

Non-Suicidal Self-Injury

$134(40.4)$

Communication of suicidality to others before the person died

$34(10.2)$

$14(35) \quad 148(39.8)$

$40(10.8)$

$109(32.8)$

$6(15) \quad 40(10.8)$

Note/message left to be found by others after the person died

$89(26.8)$

$13(32.5)$

$122(32.8)$

$83(25)$
$181(54.5)$

$\geq 1$ Suspected

$\geq 1$ Diagnosis

$120(36.1)$

7 (17.5)

$\geq 2$ Diagnoses

Diagnosed physical health conditions, ${ }^{\mathrm{d}} n(\%)$

$136(41)$

$23(57.5)$

105 (28.2)

90 (24.2)

$204(54.8)$

140 (37.6)

$\geq 2$ Diagnoses

$66(19.9)+3(75)$

$150(40.3)$

Service contact in the year before death, ${ }^{e} n(\%)$

$\geq 1$ Service

$173(52.1)$

$3(7.5)$

$69(18.5)$

$173(52.1)-21(52.5)$

$94(28.3) \quad 16(40)$

Recent GP contact (within 3 months before death)

$43(13)$

$21(52.5) \quad 194(52.2)$

$\begin{array}{ll}-6(15) & 110(29.6)\end{array}$

Refused support from service(s) $)^{\dagger}$

Psychosocial risk markers, $\mathrm{g}(\%)$

$\geq 1$ Psychosocial risk marker(s)

278 (83.7)

$6(15)$

$246(74.1)$

$34(85)$

49 (13.2)

$20(5.4)$

a. Evidence of autism: sum of 'possible diagnosis' + 'strong evidence' + 'definite diagnosis'.
b. Risk markers: sum of suicidality and self-harming behaviours, diagnosed mental and hyysical health conditions, and psychosocial risk markers.

$28(70) \quad 312(83.9)$

$274(73.7)$

$\begin{array}{lll}\chi^{2} & d & t(370) \\ - & 0.08 & -0.434\end{array}$

0.66

$0.428 \quad-\quad \quad-\quad 0.8(0.4-1.57) \quad 0.513$

$\begin{array}{llll}0.843 \quad-\quad & - & 1.55(0.61-3.95) & 0.359 \\ 0.002 & - & - & 0.98(0.49-1.98)\end{array}$

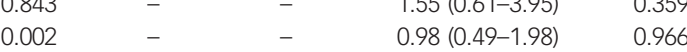

$0.002-5-300$

$1.09 \quad-\quad \quad-\quad 0.64(0.27-1.49) \quad 0.295$

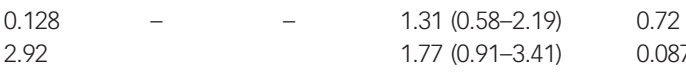

c. Diagnosed and suspected mental health conditions: depression, anxiety, personality disorder, bipolar disorder, psychosis, obsessive-compulsive disorder, post-traumatic stress disorder, social anxiety, eating disorder, attention deficit hyperactivity disorder, Tourette's

syndrome.
d. Diagnosed physical health conditions, migraines, hypothyroidism, unexplained pain, drug/alcohol dependency, liver disease, anaemia, asthma, gout, hepatitis, heart condition, high blood pressure, stress, insomnia, skin condition, cancer, chronic obstructive pulmonary disease, diabetes, chronic back pain, kidney disease/fallure, arthit is, irritable bowel syndrome, degenerative neurological condition, brain injury, epilepsy, stroke, eye problem, ear problem, genetic disorder, multiple sclerosis

practitioner (family doctor), community psychiatric nurse, hospital, drug/alcohol team, crisis team, Improving Access to

f. Refused support from service(s): the person who died refused to engage with the service they were referred to.
g. Psychosocial risk markers: socially isolated, isolated from family, alcohol/drug abuse, gambling addiction, bereavement, trauma/abuse, unstable family life; exposure to others' suicidal behaviour, lived alone, homeless, legal problems, not in employment or education,
agoression/agitation, stress, deteriorating/poor general health, chronic pain, retired, financial problems, religious, carer for another person. 
$.56-2.51)$, or evidence of autism $\left(\chi^{2}=0.94, P=0.33\right.$; OR $=1.69,95 \%$ CI 0.58-4.93). However, the people who died whose next of kin were invited to participate in stage 2 were significantly older at the time of their death (mean age 49.24 years, s.d. $=19.05$ ) than the people who died whose next of kin we were not able to invite to take part in stage 2 (mean age 42.68 , s.d. $=15.34)(t=2.53(160), P=0.018)$. In total, $29(25 \%)$ next of kin took part in the interviews (mean age 57.86 years, s.d. $=13.62,11$ male, 18 female) .

\section{Measures}

Evidence of autism

The checklist for evidence of autism developed for the analysis of the coroners' inquest records in stage 1 was adapted for the stage 2 interviews (see Appendix).

\section{Psychological autopsy interview}

Signs of possible autism in the psychological autopsy interview were required in at least two areas (using the same criteria as in the analysis of coroners' inquest records; Appendix). The interviewers (S.C., H.C.-W., S.A.-Y., A.R.) were all involved in data entry in stage 1 of the study. However, H.C.-W., S.A.-Y.and A.R. did not read the coroners inquests before the interviews. S.C. did participate in data entry of some coroners' inquests prior to the interviews, with a gap of at least 6 months between reading the records and conducting the interviews. Two interviewers were present at each interview. Both interviewers discussed evidence for autism directly after the interview was completed. Agreement was required by both interviewers to code evidence of autism, otherwise a code of 'no evidence' was returned.

\section{Social Responsiveness Scale}

The Social Responsiveness Scale - second edition (SRS-2) is a 65item proxy report rating scale of autistic traits. ${ }^{28}$ Each item is scored on a scale from 0 (never true) to 3 (always true), with higher scores indicating a greater number and severity of autistic traits. Scores at or above 60 suggest autistic traits in the range of clinical concern for possible autism. ${ }^{28}$ The SRS- 2 wording was adapted to enable next of kin to report on the behaviour of the person within the 6-month period before they died.

\section{Autism Diagnostic Interview}

The Autism Diagnostic Interview - revised (ADI-R) is a widely used and validated tool for diagnosis of autism in children and adults. ${ }^{29}$ The ADI-R is a semi-structured interview with an informant who reports on the early developmental history and/or current behaviour of the person being assessed. ${ }^{29}$ The ADI-R was modified to enable next of kin to report on the early developmental history and/or more recent behaviour present within the 6-months prior to the person's death. ADI-R interviews were conducted by trained interviewers (S.C., S.A.-Y., A.R.), with $18 \%$ of the ADI-R interviews independently scored by two interviewers. Agreement was above the required $80 \%$ (83-93\%).

\section{Procedure}

The coroners' cohort comprised next of kin of those who died, each of whom received a postal invitation through the coroners' office of site 2. Participants were invited to take part in an interview study aiming to understand why people take their own lives, to help prevent future deaths. Participants were informed that the study was exploring a range of characteristics of the person who died, including their demographics and medical information, social skills and early development. In order to minimise bias, the invitations to the coroners' cohort did not mention autism or autistic traits.
Interviews took place either in participants' homes, or on university premises, according to their preference. Prior to the interview, all participants discussed their invitation with a member of the research team either by phone or by email, to ensure they had appropriate support during and after the interview. Participants all completed the first interview, which included the psychological autopsy interview and SRS-2. If the person who died showed evidence of autism in the analysis of the coroners' records, psychological autopsy interview or SRS-2, the next of kin were invited to complete the ADI-R in a follow-up interview.

\section{Analysis}

Point biserial correlations were calculated between the SRS- 2 total scores, evidence of autism from the coroners' inquest records, evidence of autism from the psychological autopsy interviews, and whether the person who died met the threshold for autism on the ADI-R. Chi-square analyses were used to compare the occurrence of total evidence of autism (possible/strong/definite) to the prevalence of autism expected in the general alive population.

\section{Results}

\section{Stage 1: coroners' inquest records}

\section{Descriptive statistics}

Table 1 shows evidence of autism in those who died by self-harm or likely suicide from analysis of the coroners' inquest records. There was no significant difference in total evidence of autism (possible/ strong/definite) between those who likely died by suicide (11.9\%) or by self-harm without clear evidence of intent $(4.8 \%)\left(\chi^{2}=2.71\right.$, $P=0.1 ; \mathrm{OR}=2.67,95 \%$ CI $0.79-8.94)$. There were no significant gender differences in the rate of death by self-harm $(85.5 \%$ male $)$ or likely suicide $(78.4 \%$ male $)\left(\chi^{2}=1.6, P=0.21 ; \mathrm{OR}=1.62,95 \%\right.$ CI $0.76-3.46)$, or in those with $(85 \%$ male) or without $(78.9 \%$ male) any evidence of autism $\left(\chi^{2}=0.813, P=0.37\right.$; OR $=0.66$, 95\% CI 0.27-1.64).

A three-way ANOVA showed no significant differences in age according to gender $\left(F(1,363)=1.2, P=0.274, \eta_{p}{ }^{2}=0.003\right)$, evidence of autism $\left(F(1,363)=0.886, P=0.347, \eta_{p}{ }^{2}=0.002\right)$ or cause of death $\left(F(1,363)=0.335, P=0.56, \eta_{p}{ }^{2}=0.001\right)$, and no significant interactions between the variables (all $P>0.33, \eta_{p}{ }^{2}<0.003$ ). Therefore, in subsequent analyses data were combined across cause of death (self-harm/suicide), gender (male/female) and age.

\section{Evidence of autism}

Chi-square analysis showed that the proportion of individuals with total evidence of autism (possible/strong/definite; 10.8\%) was significantly higher in those who died by self-harm or suicide than the $1.1 \%$ prevalence expected in the UK general alive population ( $1.1 \%$ including people with previously unknown (i.e. undiagnosed) cases of autism $)^{3}\left(\chi^{2}=31.31, P<0.001\right.$; OR $=11.08$, 95\% CI $3.92-$ $31.31)$. In contrast, the proportion of individuals who died with a diagnosis of autism recorded $(0.5 \%)$ was not significantly different to the expected prevalence of autism in the UK general alive population $(1.1 \%)\left(\chi^{2}=0.672, P=0.41 ; \mathrm{OR}=0.5,95 \% \mathrm{CI} 0.9-2.73\right)$.

\section{Risk markers}

Table 2 shows results of the content analysis, comparing the characteristics and risk markers stratified by evidence of autism. There were no significant differences between those with evidence of autism (possible/strong/definite) and those without evidence of autism in regard to suicide method $\left(\chi^{2}=10.32, P=0.24 ; \phi=0.17\right)$. There were no significant differences in the rate of suicidality and 
self-harming behaviour, mental or physical health conditions or service contact, or psychosocial risk markers between the two groups.

\section{Stage 2: interviews with next of kin}

Evidence of autism

In total 5 (17.2\%) of the follow-up sample had possible/strong evidence of autism identified from the coroners' records in stage 1 of the study (but none had a definite diagnosis of autism recorded). The psychological autopsy interview/SRS-2 identified evidence of 'possible autism' in an additional 7 (24.1\%), giving a total of 12 (41.4\%) individuals. However, 'strong evidence of autism' was not confirmed, as none of the 12 met the threshold for autism on the ADI-R.

Chi-square analysis showed that the number of individuals with possible autism in the coroners' cohort $(n=12,41.4 \%)$ was significantly higher than expected in the general alive population $(1.1 \%)$ $\left(\chi^{2}=12, P<0.001 ;\right.$ OR $=19.76,95 \%$ CI $\left.2.36-165.84\right)$.

\section{Reliability}

SRS-2 scores were not significantly correlated with evidence of autism from the psychological autopsy interview using point biserial correlation $\left(n=29 ; r_{\mathrm{pb}}=0.259, P=0.176\right)$, or with evidence of autism identified from the coroners' inquest records using spearman's correlation $\left(n=29 ; r_{\mathrm{s}}=0.06, P=0.754\right)$.

\section{Discussion}

\section{Main findings}

As far as we know, this is the first study to examine evidence of autism and autistic traits in those who died by suicide in England. Through analysis of 372 coroners' inquest records in two regions of England, there was evidence of autism and elevated autistic traits in $10.7 \%$ of those who died by suicide - 11 times higher than prevalence of autism in the UK general population similarly including previously unknown diagnoses of autism (1.1\%). ${ }^{3}$ Given that coroners' records do not systematically gather evidence of autism and autistic traits, this is likely an underestimate of the true rate.

Consistent with this, subsequent interviews with a subsample of the next of kin of those who died showed evidence of elevated autistic traits indicating possible autism in $41.4 \%$ of the sample. However, none of the follow-up sample showed strong evidence of undiagnosed autism using a validated diagnostic instrument (ADI-R). This high rate (41.4\%) of possible autism identified in the current study is consistent with previous research in which $40.6 \%$ of those who attempted suicide (without autism or suspected autism diagnosis) scored above the cut-off for clinical concern for possible autism on the Autism Spectrum Quotient; when including people with autism and those with suspected autism, 53.9\% scored above the cut-off. ${ }^{13}$ Results suggest that autistic traits in the range of clinical concern for possible autism (regardless of confirmed diagnosis) are also significantly over-represented in those who die by suicide.

Analysis of the coroners' records showed a similarly high number of risk markers in those who died with evidence of autism compared with those who died without evidence of autism. There was also a broadly similar pattern of risk markers between these groups across both stages of the study. Therefore, the current research emphasises common collective concerns for the prevention of suicide, regardless of evidence of autism. Suicide method did not significantly differ between the groups, contrary to previous research suggesting that people with autism may use more lethal forms of attempting suicide. ${ }^{30}$

The most commonly shared characteristics of those who died included previous self-harm and/or suicide attempt, at least one diagnosed or undiagnosed physical or mental health problem, poor current engagement with services and psychosocial risk markers (for example financial difficulties, recent unemployment, bullying and abuse, bereavement, relationship breakdown, living alone and/or recent changes to living situation, exposure to others' suicidal behaviour and social isolation).

Those who died did not typically experience one risk marker in isolation, but a number of different risk markers (on average $n=6$ identified from the coroners' records). This is consistent with previous suicide research and theory showing that the accumulation of background risk factors (personal and historical events) in the context of current stressors increases risk of attempting suicide. ${ }^{16}$ There was limited evidence of different or unique risk markers for suicide in those with evidence of autism or elevated autistic traits.

\section{Strengths and limitations}

The current study has a number of strengths and limitations. Limitations include the relatively small subsample of next of kin and those with evidence of autism identified in the study. A large number of comparisons were carried out, and, although a conservative alpha level was used in the study, many comparisons were underpowered. Although all proxy report measures used here demonstrate evidence of validity, undiagnosed conditions cannot be confirmed, as it was not possible to assess the person who died directly. The team who conducted the interviews with next of kin were also involved with data entry from the coroners' records, which could have led to some bias. However, this was minimised by three interviewers only inputting data from the coroners' records after the interviews, and one interviewer having a gap of at least 6 months between inputting data from the coroners' records and conducting the interviews.

Although analysis of the coroners' records covered two regions of England, next of kin were only invited from one of the coroner's offices covering one region of England, limiting the generalisability of results. The characteristics of those who died included in stage 2 interviews with their next of kin, also tended to be slightly older than those who died where we were not able to contact next of kin to participate in the second stage of the study. A majority of next of kin who took part in the interviews were female, which could also limit the generalisability of the results from the follow-up interviews.

Reliability analysis showed that scores on a validated autism screening tool (SRS-2) did not significantly correlate with evidence of autism identified from the coroners' records. Strong evidence of possible undiagnosed autism identified from the coroners' records was also not confirmed using a validated autism diagnostic tool (ADI-R). The interviews with next of kin identified evidence of autism or elevated autistic traits in an additional seven people who died. This suggests that gathering evidence of autism from the coroners' records alone is not valid, and it is important to interview next of kin to identify further evidence of autism and autistic traits.

Strengths of the study include that a range of sources of evidence for autism and autistic traits were systematically gathered, including validated autism screening (SRS-2) and an autism diagnostic tool (ADI-R). Interrater reliability was used extensively to ensure high validity and consistency of data classification and coding.

\section{Implications}

There are clear implications for suicide prevention. First, as elevated autistic traits and possible undiagnosed autism are over- 
represented in those who attempt and die by suicide, it is crucial for clinicians to screen for autistic traits and possible undiagnosed autism in patients presenting with suicidal thoughts and behaviours, and to offer appropriate tailored support. ${ }^{31}$ A quick (10-item) and validated screening instrument for autistic traits is the Autism Spectrum Quotient-10, ${ }^{32}$ although the full Autism Spectrum Quotient does not take much longer to complete $(50$ items $)$ and would provide more detail. ${ }^{12}$ Scoring above cut-off on either version should support a referral for an autism assessment.

Second, although $32 \%$ of the sample communicated their suicidal thoughts to another person before they died, some families reported how the death of their loved one 'came out of the blue' with no obvious warning signs. This suggests that some people do not present with clear warning signs or risk markers for suicide, but nevertheless may be at significant risk of suicide. It is therefore important to ask whether a person is feeling suicidal, and to believe the person if they disclose feeling suicidal. ${ }^{33}$ Clinicians should also regularly check up on patients, as risk can change dramatically in a short period of time.

Third, given that the current study suggests that evidence of autism is typically not included in coroners' inquests in England, it is crucial for coroners to begin to systematically gather evidence of autism and autistic traits in inquests. This will enable more accurate nationwide estimates of autism and autistic traits in those who die by suicide in the UK to be determined, and may facilitate identification of potentially unique suicide prevention targets identified in this group. This could be achieved by asking general practitioners to confirm whether the person who died had an autism diagnosis or suspected autism, and/or by asking next of kin to complete a brief checklist to identify autistic traits in the person who died.

Finally, in terms of suicide prevention programmes, it is important that these are tailored to autistic people. The top autism community priority for suicide prevention is removing barriers to support and treatment, ${ }^{33}$ given that autistic people report being excluded from services, and receiving inappropriate support and treatment for mental health problems and suicidality. ${ }^{31,33}$ Our results also suggest that suicide prevention programmes need to tackle wider societal issues, such as social exclusion and isolation, poverty, unemployment, trauma and abuse. ${ }^{6,8,19}$ All of these are significantly more common experiences among autistic people compared with non-autistic people, and increase their risk of attempting suicide. $^{5}$

Suicide prevention programmes could also help the person understand how an exclusive focus on one's own point of view might lead a person to make incorrect assumptions about other people's thoughts and feelings (for example believing incorrectly that 'no one cares about me, the world would be better off without me, and my death would not affect anyone else'), leading them to consider suicide. Programmes could also explain and demonstrate that with the right support (peer support, support from social care services, and supported employment and befriending schemes), quality of life can be improved and social isolation can be reduced.

In conclusion, our results suggest that autism and autistic traits are over-represented in those who died by suicide in England; $41.4 \%$ of those who died had evidence of elevated autistic traits. This is consistent with a growing body of research showing that autistic people, and those with elevated autistic traits, are at increased risk of contemplating, attempting and dying by suicide. ${ }^{5}$ It is imperative for clinical services to address barriers to treatment and support that are experienced by autistic people, and to develop programmes with and for autistic people to reduce their high risk of suicide. ${ }^{33}$

\section{Data availability}

The data that support the findings of this study are available from the corresponding author (S.C.), on reasonable request. The data are not publicly available owing to their containing information that could compromise the privacy of research participants.

Sarah Cassidy (1D, School of Psychology, University of Nottingham, UK and Faculty of Health and Life Sciences, Coventry University, UK; Sheena Au-Yeung, School of

Psychology, University of Nottingham, UK, Faculty of Health and Life Sciences, Coventry University, UK and Department of Psychiatry, University of Oxford, UK;

Ashley Robertson, Faculty of Health and Life Sciences, Coventry University, UK and School of Psychology, University of Glasgow, UK; Heather Cogger-Ward, School of Psychology, University of Nottingham, UK, School of Psychology, University of Lincoln, UK and Lincolnshire Partnership NHS Foundation Trust, UK; Gareth Richards, School of Psychology, Newcastle University, UK and Autism Research Centre, Department of Psychiatry, University of Cambridge, UK; Carrie Allison, Autism Research Centre, Department of Psychiatry, University of Cambridge, UK; Louise Bradley, The International Centre, University of Bedfordshire, UK; Rebecca Kenny, Autism Research Centre, Department of Psychiatry, University of Cambridge, UK; Rory O'Connor $\mathbb{D}_{\text {, }}$ Suicidal Behavior Research Laboratory, Institute of Health \& Wellbeing, University of Glasgow, UK; David Mosse, SOAS, University of London, UK; Jacqui Rodgers, Population Health Sciences Institute, Sir James Spence Institute, Newcastle University, UK; Simon Baron-Cohen, Autism Research Centre, Department of Psychiatry, University of Cambridge, UK

Correspondence: Sarah Cassidy. Email: sarah.cassidy@nottingham.ac.uk

First received 25 May 2021, final revision 11 Jan 2022, accepted 17 Jan 2022

\section{Acknowledgements}

We sincerely thank the families and friends of those who died who took part in this study to help us to understand and prevent suicide, and all the staff in the coroners' offices who supported this research project. We are also extremely grateful to those with lived experience and expertise who provided feedback on the interview schedules and participant recruitment materials for the current study. We additionally thank Professor Clare Wood, Professor Erica Bowen, Susan Goodlad, Dr Rebecca Shaw, Dr Rosie Holt and Dr Sarah Griffiths, for valuable discussions, and Claire Pillinger, Dr Penelope Hannant, Kathy Cook, Gurkaran Dhanda, Becky Cooke and Sam Waldron for their assistance with data entry in stage 1 of the study.

\section{Author's contributions}

S.C., S.B.-C. and C.A. conceived of the study. All authors contributed to the study design. S.C., G.R., L.B., H.C.-W., S.A.-Y., A.R. all contributed to data collection. S.C. and H.C.-W. contributed to data analysis. All authors contributed to data interpretation. S.C. wrote the first draft of the manuscript. All authors provided critical feedback on the draft manuscript, and read and approved the manuscript before submission for publication.

\section{Funding}

This research was supported by Autistica [grant number: 7247], the Economic and Social Research Council Future Research Leaders grant [grant number: ES/N000501/2], Coventry University, and the University of Nottingham (received by S.C.). S.B.C. received funding from the Wellcome Trust 214322 \Z\18VZ. For the purpose of Open Access, the author has applied a CC BY public copyright licence to any Author Accepted Manuscript version arising from this submission. In addition, S.B.-C. received funding from Innovative Medicines Initiative 2 Joint Undertaking (JU) under grant agreement No 777394 . The JU receives support from the European Union's Horizon 2020 research and innovation programme and EFPIA and AUTISM SPEAKS, Autistica, SFARI. S.B.-C. also received funding from the Autism Research Trust, SPEAKS, Autistica, SFARI. S.B.-C. also received funding from the Autism Research Trust,
SFARI, the Templeton World Charitable Fund, SFARI and the NIHR Cambridge Biomedical Research Centre. The research was supported by the National Institute for Health Research (NIHR) Collaboration for Leadership in Applied Health Research and Care East of England at Cambridgeshire and Peterborough NHS Foundation Trust. The views expressed are those of the author(s) and not necessarily those of the NHS, NIHR or Department of Health and Social Care. The funding sources were not involved in any aspect of the study design, recruitment, data collection, analysis, interpretation of results, writing of the manuscript, or the decision to submit for publication. The corresponding author has full access to all data used in the study and had final responsibility for the decision to submit for publication.

\section{Declaration of interest}

The authors disclose no conflicts of interest. 


\section{Checklist for gathering evidence of autism from the coroners' inquest records/psychological autopsy interview, and stage 2 interviews}

\begin{tabular}{|c|c|c|}
\hline $\begin{array}{l}\text { Evidence of } \\
\text { autism }\end{array}$ & $\begin{array}{l}\text { Definition from coroners' inquest records / psychological autopsy } \\
\text { interview }\end{array}$ & Definition from stage 2 interviews \\
\hline $\begin{array}{l}\text { Definite } \\
\quad \text { diagnosis }\end{array}$ & $\begin{array}{l}\text { Clinical diagnosis of autism noted in the coroners' inquest record/ } \\
\text { psychological autopsy interview }\end{array}$ & $\begin{array}{l}\text { Clinical diagnosis of autism noted in the coroners' inquest record } \\
\text { and/or psychological autopsy interview }\end{array}$ \\
\hline $\begin{array}{l}\text { Strong } \\
\quad \text { evidence }\end{array}$ & $\begin{array}{l}\text { Possible undiagnosed autism noted in the coroners' inquest record/ } \\
\text { psychological autopsy interview, and clear indicators in at least two } \\
\text { of the following areas: } \\
\text { (a) social/communication difficulties; (b) narrow interests; (c) } \\
\text { routines; (d) sensory difficulties; (e) special educational needs in } \\
\text { childhood }\end{array}$ & $\begin{array}{l}\text { Clear indicators of undiagnosed autism on at least one of the } \\
\text { sources of evidence (coroners' record, psychological autopsy } \\
\text { interview, SRS-2 cut-off), and met the clinical cut-off for autism on } \\
\text { the ADI-R }\end{array}$ \\
\hline $\begin{array}{l}\text { Possible } \\
\text { diagnosis }\end{array}$ & $\begin{array}{l}\text { Clear indicators of elevated autistic traits indicating clinical concern for } \\
\text { possible autism in at least two areas (as above), but possible autism } \\
\text { diagnosis not noted in the coroners' inquest record/psychological } \\
\text { autopsy interview }\end{array}$ & $\begin{array}{l}\text { Clear indicators of elevated autistic traits indicating clinical concern } \\
\text { for possible autism on at least one of the sources of evidence } \\
\text { (coroners' record, psychological autopsy interview, SRS-2 cut- } \\
\text { off), but did not meet the clinical cut-off for autism on the ADI-R }\end{array}$ \\
\hline No evidence & $\begin{array}{l}\text { No clear indicators of autism in the coroners' inquest record/ } \\
\text { psychological autopsy interview }\end{array}$ & $\begin{array}{l}\text { No clear indicators of autism on any of the sources of evidence } \\
\text { (coroners' record, psychological autopsy interview, SRS- } 2 \text { cut-off) }\end{array}$ \\
\hline
\end{tabular}

\section{References}

1 American Psychiatric Association. Diagnostic and Statistical Manual of Mental Disorders (DSM-5). American Psychiatric Association, 2013.

2 Lai MC, Baron-Cohen S. Identifying the lost generation of adults with autism spectrum conditions. Lancet Psychiatry 2015; 2: 1013-27.

3 Brugha TS, Spiers N, Bankart J, Cooper SA, McManus S, Scott FJ, et al. Epidemiology of autism in adults across age groups and ability levels. $\mathrm{Br} \mathrm{J}$ Psychiatry 2016; 209: 498-503

4 Hannant P, Cassidy S, Tavassoli T, Mann F. Sensorimotor difficulties are associated with the severity of autism spectrum conditions. Front Integr Neurosci 2016; 10: 28

5 Cassidy S. Suicidality and self-harm in autism spectrum conditions. In oxford Handbook of Autism and Co-Occurring Psychiatric Conditions (eds S White, B Maddox, C Mazefsky): 349-68. Oxford University Press, 2020

6 Griffiths S, Allison C, Kenny R, Holt R, Smith P, Baron-Cohen S. The vulnerability experiences quotient (VEQ): a study of vulnerability, mental health and life satisfaction in autistic adults. Autism Res 2019; 12: 1516-28.

7 Cassidy S, Bradley P, Robinson J, Allison C, McHugh M, Baron-Cohen S. Suicidal ideation and suicide plans or attempts in adults with Asperger's syndrome attending a specialist diagnostic clinic: a clinical cohort study. Lancet Psychiatry 2014; 1: 142-7.

8 Pelton MK, Crawford H, Robertson AE, Rodgers J, Baron-Cohen S, Cassidy S. Understanding suicide risk in autistic adults: comparing the interpersonal Theory of Suicide in autistic and non-autistic samples. J Autism Dev Disord 2020: 10: 3620-37.

9 Takara K, Kondo T. Comorbid atypical autistic traits as a potential risk factor for suicide attempts among adult depressed patients: a case-control study. Ann Gen Psychiatry 2014; 13: 33

10 Rydén G, Rydén $\mathrm{E}$, Hetta J. Borderline personality disorder and autism spectrum disorder in females: a cross-sectional study. Clin Neuropsychiatry 2008; 5: $22-30$

11 South M, Beck JS, Lundwall R, Christensen M, Cutrer EA, Gabrielsen TP, et al. Unrelenting depression and suicidality in women with autistic traits. J Autism Dev Disord 2020; 50: 3606-19.

12 Baron-Cohen S, Wheelwright S, Skinner R, Martin J, Clubley E. The autismspectrum quotient (AQ): evidence from Asperger syndrome/high-functioning autism, males and females, scientists and mathematicians. J Autism Dev Disord 2001; 31: 5-17.

13 Richards G, Kenny R, Griffiths S, Allison C, Mosse D, Holt R, et al. Autistic traits in adults who have attempted suicide. Mol Autism 2019; 10: 26.

14 Hirvikoski T, Mittendorfer-Rutz E, Boman M, Larsson H, Lichtenstein P, Bölte S Premature mortality in autism spectrum disorder. Br J Psychiatry 2016; 208 232-8.
15 Kirby AV, Bakian AV, Zhang Y, Bilder DA, Keeshin BR, Coon H. A 20-year study of suicide death in a statewide autism population. Autism Res 2019; 12: 658-66.

16 O'Connor RC, Kirtley OJ. The integrated motivational-volitional model of suicidal behaviour. Philos Trans R SOC B Biol Sci 2018; 373: 20170268.

17 Hull L, Mandy W, Lai MC, Baron-Cohen S, Allison C, Smith P, et al. Development and validation of the camouflaging autistic traits questionnaire (CAT-Q). J Autism Dev Disord 2019; 49: 819-33.

18 Cassidy SA, Gould K, Townsend E, Pelton M, Robertson AE, Rodgers J. Is camouflaging autistic traits associated with suicidal thoughts and behaviours? Expanding the interpersonal psychological theory of suicide in an undergraduate student sample. J Autism Dev Disord 2020; 50: 3638-48.

19 Mitchell P, Sheppard E, Cassidy S. Autism and the double empathy problem: implications for development and mental health. Br J Dev Psychol 2021; 39: 1-8.

20 Cassidy SA, Bradley L, Cogger-Ward H, Shaw R, Bowen E, Glod M, et al. Measurement properties of the suicidal behaviour questionnaire-revised in autistic adults. J Autism Dev Disord 2020; 50: 3477-88.

21 Cassidy SA, Bradley L, Cogger-Ward H, Rodgers J. Development and validation of the suicidal behaviours questionnaire-autism spectrum conditions in a community sample of autistic, possibly autistic and non-autistic adults. $\mathrm{Mol}$ Autism 2021; 12: 1-22.

22 Conner KR, Beautrais AL, Brent DA, Conwell Y, Phillips MR, Schneider B. The next generation of psychological autopsy studies: Part I. interview content Suicide Life Threat Behav 2011; 41: 594-613.

23 Hawton K, Appleby L, Platt S, Foster T, Cooper J, Malmberg A, et al. The psychological autopsy approach to studying suicide: a review of methodological issues. J Affect Disord 1998; 50: 269

24 Appleby L, Turnbull P, Kapur N, Gunnell D, Hawton K. New standard of proof for suicide at inquests in England and Wales. Br Med J 2019; 366: 14745.

25 Rockett IR, Kapusta ND, Coben JH. Beyond suicide: action needed to improve self-injury mortality accounting. JAMA PSychiatry 2014; 71: 231-2.

26 Palmer BS, Bennewith O, Simkin S, Cooper J, Hawton K, Kapur N, et al. Factors influencing coroners' verdicts: an analysis of verdicts given in 12 coroners' districts to researcher-defined suicides in England in 2005. J Public Health 2015; 37: 157-65.

27 World Health Organization. The ICD-10 Classification of Mental and Behavioural Disorders: Diagnostic Criteria for Research. World Health Organization, 1993

28 Constantino JN, Gruber CP. Social Responsiveness Scale: SRS-2. Western Psychological Services, 2012.

29 Rutter M, LeCouteur A, Lord C. Autism Diagnostic Interview-Revised Manual. Western Psychological Services, 2003.

30 Kato K, Mikami K, Akama F, Yamada K, Maehara M, Kimoto K, et al. Clinical features of suicide attempts in adults with autism spectrum disorders. Gen Hosp Psychiatry 2013; 35: 50-3. 
31 Camm-Crosbie L, Bradley L, Shaw R, Baron-Cohen S, Cassidy S. 'People like me don't get support': autistic adults' experiences of support and treatment for mental health difficulties, self-injury and suicidality. Autism 2019; 23: 1431-41.

32 Allison C, Auyeung B, Baron-Cohen S. Toward brief "red flags" for autism screening: the Short Autism Spectrum Quotient and the short quantitative checklist in 1,000 cases and 3,000 controls. J Am Acad Child Adolesc Psychiatry 2012; 51: 202-12.
33 Cassidy S, Goodwin J, Robertson A, Rodgers R. INSAR Policy Brief: Autism Community Priorities for Suicide Prevention. International Society for Autism Research, 2021 (https://cdn.ymaws.com/www.autism-insar.org/resource/ resmgr/files/policybriefs/2021-insar_policy_brief.pdf).

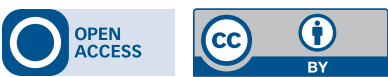

\section{Psychiatry in History}

\section{Reascending the magic mountain: Thomas Mann's Dr Krokowski and modern psychiatry}

\section{Alexander Smith (iD) and Michael Liebrenz}

On a recent trip to Davos, Switzerland, for a forensic psychiatric evaluation, we marvelled at the majestic Schatzalp landscape and recalled the complexities of Thomas Mann's The Magic Mountain (1924). ${ }^{1}$ Alongside the eponymous sanatorium-turnedhotel, Mann's bildungsroman was set atop this alpine peak in Switzerland. The book follows Hans Castrop's convalescence and subsequent education about wide-ranging, innovative dialectics that intrigued contemporaneous European medical circles.

Particularly illuminating is the attending psychiatrist, Dr Edhin Krokowski, who instantiates elements of Georg Groddec, Richard von Krafft-Ebing and Sigmund Freud. An 'idealist of the pathological', Krokowski considers psychiatric symptoms 'a disguised manifestation of the power of love', instead emphasising the inorganic basis of mental disorders, or 'the bringing of light into the unconscious mind'. ${ }^{1}$ Mann ironises such ideas by conveying Krokowski's occultist descent.

For us, the author focused on these concepts precisely because they exemplified state-of-the-art psychiatric thoughts of the time. Sharing similar biographies, Mann and Freud corresponded regularly, but Mann disputed Freudian tenets. Contemplating the schatzalp today and recalling this fictional physician, we speculated on the hypothetical (and admittedly frivolous) notion of a modern-day Krokowski, and through him, which novel psychiatric ideas Mann would dissect.

With its recent technological developments, Mann may have depicted his archetype as a proponent of biological psychiatry. At one juncture, Castorp reacts to an x-ray of his cousin's heart (cutting-edge technology in this epoch); he was 'moved', but experienced 'uneasy undoubt, as to whether it was really permissible and innocent to stand here [ ... ] and gaze'. ${ }^{1}$ The protagonist's fascination is tempered by morbid unease, foreshadowing some debates about the contexts in which neuroimaging should be utilised.

Perhaps a contemporary Krokowski may embody principles of social psychiatry? This would depart from his refusal to 'deduce the perversity of the whole from [ ... ] its parts'. ${ }^{1}$ One could imagine Mann appraising psychiatric advancements by having Krokowski discuss social determinants. Equally, Mann may have undermined this by symbolising the tension between prevention and treatment previously illustrated by detractors of social psychiatry.

Given its predominance, a contemporary Krokowski could promote the biopsychosocial concept; the eclecticism of this has been criticised as enabling psychiatrists to 'pick and choose' their principles of care. Castorp seemingly rejects Krokowski's latter occultism, yet he ultimately disavows his recent insights into rational dialectics by enlisting during the First World War 'under pressure from the prevailing temper'. ${ }^{1}$ Consequently, even today, Mann may still have foregrounded Krokowski's psychodynamic approaches, exploiting the purported flexibility of the biopsychosocial model.

Of course, this represents an arbitrary and light-hearted dialogue on a train ride from Davos; others will undoubtedly (and justifiably) have divergent opinions. Nonetheless, if Mann were writing now, his critical ambivalence may have been piqued by these or other psychiatric concepts, the advantages and disadvantages of which have been extensively debated in scientific literature. Less trivially, the boundlessness of the Schatzalp reminds us how far psychiatry has come since Mann raised his pen, and how much further our discipline can go. The possibilities are as vast as the magic mountain we beheld.

\section{Declaration of interest}

None.

\section{Reference}

1 Mann T. The Magic Mountain (translator HT Lowe-Porter). Vintage Books, 1999.

(c) The Author(s), 2022. Published by Cambridge University Press on behalf of the Royal College of Psychiatrists 